\title{
ENFOQUES DE ESTUDIO DE CASOS EN LA INVESTIGACIÓN DE ENFERMERÍA
}

\section{CASE STUDY APPROACHES IN NURSING RESEARCH}

\author{
Eugenia URra Medina* \\ Rocío Núñez Carrasco ${ }^{* *}$ \\ CARMEn Retamal VALENZUEla** \\ LUCY JURE CARES ${ }^{* * *}$
}

\begin{abstract}
RESUMEN
Los estudios de casos han sido usados en una variedad de disciplinas en las ciencias sociales y salud, al tener cualidades para comprender en profundidad un fenómeno en variados contextos y situaciones naturales. Sin embargo, su uso ha sido confuso por las diferentes visiones de los investigadores. Este artículo tiene como propósito hacer una distinción de los estudios de casos: con perspectiva cualitativa y como un diseño de investigación. Los estudios de casos cualitativos se originan por la forma particular de ver el caso como un todo: su contexto y sus límites, con análisis intensivo del caso o casos colectivos, y siempre bajo la concepción de su idiosincrasia y sin generalización. El diseño de estudios de casos como parte de una estrategia investigativa busca dar respuesta a una pregunta de investigación que permite usar diferentes métodos para hacer constantes comparaciones múltiples. En síntesis, los estudios de casos son usados en ambas formas por los investigadores y tienen un potencial de utilidad en situaciones y contextos de enfermería y salud.
\end{abstract}

Palabras clave: Estudio de casos, investigación en enfermería, investigación cualitativa.

\begin{abstract}
Case studies have been used into social sciences and health disciplines because of their properties to understand complex phenomena in a variety of contexts and situations. However, its use has been confusing because of the different researcher's perspectives. This article aims to distinguish two types of two types of case studies approaches: the one with a qualitative perspective and as a design research strategy. The qualitative case study are originated by the particular way of seing the case as a whole: its contexts and limits, intensive case analysis or collective cases, and always under the conception of their idiosyncrasy without generalization. In a research design, case study is a research strategy that tries to answer a research question by applying different methods for data collection and analyzing by using constant comparison. Currently, both approaches of case studies are used by researchers, having a potential benefit for nursing and health settings and contexts.
\end{abstract}

Key words: Case studies, nursing research, qualitative research.

Fecha recepción: 22/08/12 Fecha aceptación: 31/07/13

\footnotetext{
* Enfermera. Académica Enfermería Universidad de La Serena, Chile. Email: eurra@userena.cl

${ }^{* *}$ Enfermera. Académica Enfermería, Universidad de Santiago, Chile. Email: rocio313@gmail.com

${ }^{* * *}$ Enfermera. Académica Enfermería, Universidad de La Serena, Chile. Email: cretamal@userena.cl

${ }^{* * * *}$ Enfermera. Coordinadora Centro de Salud Familiar 2 de Septiembre, Los Ángeles, Chile. Email: lucyjure@yahoo.com
} 


\section{INTRODUCCIÓN}

Los investigadores en salud, como en la disciplina de enfermería, han incursionado en los Estudios de Casos (EC) desde hace más de una década, tanto desde escenarios cuantitativos como cualitativos (1-5), lo que implica que a veces se entrecrucen perspectivas epistemológicas y metodológicas. El Estudio de Casos ha sido usado en una variedad de disciplinas como un detallado examen de un fenómeno único (caso) dentro del contexto de la vida real y consiste en una investigación en profundidad de un caso usando diferentes perspectivas y métodos para investigar los fenómenos sociales $(6,7)$. Su particularidad es que considera esencial dos cosas relevantes para entender el fenómeno: el contexto donde toma lugar y la inclusión de los diferentes elementos que podrían impugnarse en un caso particular, sea un individuo, una institución, o un grupo de casos. Johansson sintetiza al caso como "una unidad que funciona compleja, se investiga en su contexto natural con métodos múltiples, y debe ser contemporáneo" (8).

Los Estudios de Casos se originaron a inicios de 1900 en Antropología, en primera instancia, para tener después una fuerte imposición lógico-positivista dentro de las ciencias sociales, y retornó en los años 1960 en adelante en una segunda generación metodológica: entre positivismo y hermenéutica (8), teniéndose una mirada de eclecticismo y pragmatismo, tanto en las metodologías cualitativas, originadas por una construcción más conceptual como la teoría fundada y fenomenológica como también por una mirada más pragmática desde los paradigmas cuantitativos, como un método integrativo dentro de un diseño. Por esta razón, hay en la literatura una controversia en cómo los EC son seleccionados y usados por los investigadores, derivando en confusión y relativa credibilidad de los estudios.

En el proceso metodológico de conducir un EC -algunos investigadores pueden confundir la unidad de estudio (el caso) y el producto de este tipo de investigación- (9), esta situación es originada porque los investigadores tienen diferentes formas de abordarlo. Yin (10) afirma, por un lado, que los EC son un método de investigación concebido como una indagación empírica que investiga un fenómeno contemporáneo dentro de su contexto de vida real, especialmente cuando las fronteras entre fenómeno y contextos no son tan evidentes. Y agrega Yin (10) que la orientación de la pregunta de investigación qué o cómo puede dar respuesta ya sea al explicar, describir o explorar eventos en el contexto diario en que ellos ocurren, por ejemplo: explicar causas y procesos resultantes en una política o un programa nuevo. Así, respondiendo a preguntas sobre cómo y por qué de una serie de eventos contemporáneos, los EC estarán abiertos a conformar una teoría o categorías conceptuales que guiarán la investigación y el análisis de los datos. Por otro lado, Merriam (9) sostiene que los EC son una descripción holística e intensiva y analizan, ya sea, una instancia única, un fenómeno o una unidad social. Más tajante, Stake (11) afirma que el caso es estudiado en su propio derecho, sin ninguna manipulación del investigador, ya que el caso es concebido como el objeto de estudio, y agrega que para la comunidad de investigadores cualitativos el EC se concentra en un conocimiento experiencial del caso y una atención focalizada en las influencias del contexto político y social, entre otros. Es decir, el caso es único y puede referirse a un individuo, un grupo, un programa, o una organización y, cualitativamente, es una perspectiva en que el contexto y su delimitación es una entidad por sí misma: una unidad que puede ser circunscrita $(9,12)$. En general, los investigadores cualitativos concuerdan sobre que los EC estudian un caso singular, temporal, limitado en forma física o social, complejo en naturaleza, y único, y de esta forma no es comparable con otros casos $(9,12)$. Por ello, los 
investigadores más novatos (13-15) pueden enfrentarse al dilema del cómo abordar los EC y por ende en su manejo podrán incurrir en falencias, omisiones, o carencia de rigor metodológico.

Por lo expuesto, el propósito de este artículo es revisar los dos enfoques de EC, sus características y aspectos esenciales, tanto desde una perspectiva cualitativa como desde un diseño de investigación, con el fin de distinguir las dos miradas investigativas. Contribuirá a que profesionales de la salud puedan utilizarlo, ya que tiene múltiples aplicaciones para una mejor comprensión de los fenómenos situados en ambientes de salud (organización, programa, unidad), o por sus particularidades metodológicas para resolución de fenómenos relevantes en usuarios con una problemática de salud en ambientes naturales. Ciertamente, las limitaciones y restricciones de la validez y generalización implican dilemas y oportunidades para los investigadores que usan los EC que pueden ser entendidos dentro del contexto de la crisis de modernidad y de la ciencia y así afectar la forma de llevar a cabo los EC. Por ello Reyes y Hernández señalan seis puntos críticos a considerar en su aplicación: "La vía de inferencia inductiva-deductiva, la selección del caso, la determinación de la unidad de análisis, las técnicas de recolección de datos, los métodos de análisis y los criterios de calidad" (16).

\section{ANTECEDENTES DEL ESTUDIO DE CASOS}

En la literatura se observa una serie de referencias a los EC en reportes y aplicaciones de ellos, no obstante, parece haber poco entendimiento sobre qué son, cómo son usados y qué se debe analizar. Dado que no hay una simple taxonomía para que puedan ser clasificados y desarrollados, ya sea que los tipos de EC han sido descritos como estrategias de investigación, diseños de investigación, forma de reportes, técnicas de enseñanza o métodos de evaluación o por sus contextos también muy variados: colegios, centros de atención de salud, hospitales, sistema militar, negocios, industrias y han sido aplicados en varias disciplinas, tales como: psicología, antropología, historia, ética, economía, enfermería, leyes, educación, trabajo social, ciencias políticas, administración pública, entre otras (17). Y porque sus unidades de análisis son diferentes y pueden ser personas, familias, grupos, comunidades, organizaciones, cultura, eventos, movimientos, programas o procesos. Con estos antecedentes tan amplios, la metodología y diseños han incursionado desde indagaciones cualitativas naturistas puras hasta experimentos de caso único, o diseños en series de tiempo complejas.

Por tanto, según su propósito investigativo pueden ser exploratorios, descriptivos, interpretativos, o explicatorios (18). Por ejemplo, al desarrollar un EC, el investigador puede utilizarlo para realizar una crónica (registro de hechos o eventos temporales en relación a cómo ellos ocurrieron; para presentar hechos ya sea describir, caracterizar, señalar; para enseñar (dar instrucción), o para testificar (usar un caso para testear una teoría particular y/o hipótesis). Por estas cualidades, los EC han sido conducidos en diferentes niveles de complejidad desde un episodio único, breve, trivial hasta un evento de vida principal complejo con múltiples tramas.

\section{A. Los Estudios de Casos como perspectiva cualitativa}

En la literatura hay críticas a los enfoques de EC y particularmente de aquellos que tienen orientación cualitativa, en especial con la tendencia actual de la práctica basada en la evidencia, son observados como poco científicos por los temas de validez y generalización o particularmente porque en salud los profesionales de enfermería o medicina se ven más involucrados en la aplicación de reglas científicas formales y rígidas en que hay necesi- 
dad de verificar resultados empíricos, ya sea del tratamiento y cuidados de los pacientes, más que en enfatizar temas como la experiencia personal, la observación y la reflexión. Por esto, hay que tener en cuenta la instancia filosófica donde el investigador se sitúa, ya que determinará la pregunta de investigación, y no será la metodología de investigación la que define al EC sino la forma de determinar un estudio en particular. Creswell (19) afirma que la tradición de indagación en la investigación cualitativa de EC tiene una historia en alguna disciplina y así una metodología distintiva que caracteriza su enfoque. Y es aquí donde los enfoques cualitativos con un enfoque etnográfico, constructivistas o teóricos (de narrativas, entre otras) pueden despejar mejor lo que es particular de un caso único, y de este modo, la elección del caso y su estudio se determina por el interés en el mismo, mientras el diseño metodológico queda relegado a un segundo lugar $(12,20)$.

Los EC cualitativos son más comprensibles abordando el fenómeno de estudio en ambientes naturales (situación que no sucede con un diseño de investigación con una adaptación específica que recoge los datos) (20), no obstante, lo que determina un EC cualitativo será plantearse una descripción de una situación real, que está asociado con un fenómeno contemporáneo y que ha sido socializado por el grupo al cual pertenece, $y$ donde su foco no es(son) una(s) persona(s) en sí, pero sí una(s) persona(s) en una situación, institución, organización, evento o un proceso (como contexto ecológico). De este modo, se conceptualiza como una investigación sistemática e intensiva ya sea de un caso individual, comunidad o alguna unidad típicamente conducida bajo condiciones naturales, en la cual el investigador examina los datos en profundidad relacionándolos con antecedentes, situaciones comunes, características del ambiente y las interacciones.

Es decir, el EC es un estudio detallado e intensivo de un individuo o grupo como una entidad, a través de la observación, autorre- porte, entrevista, etc. De este modo, son de naturaleza sensible a la complejidad y a la historicidad específica, ya que esta estrategia subraya lo diverso y excepcional (9). Como resultado, el investigador abordará, con una profunda visión asociada a la unidad de análisis de tipo única. De este modo, los EC son considerados como una apreciación genuina y única dada por el principal axioma de este tipo investigativo: que hay variadas realidades múltiples; entonces, el caso tiene múltiples dimensiones. En resumen, los EC con tradición o perspectiva cualitativa pueden distinguirse como una investigación profunda y única de un hecho social y la unidad de análisis permanece en el objeto único, otorgando al investigador una perspectiva holística de una situación real en su propio contexto (9, 12). El EC cualitativo, entonces, requiere que su foco sea el caso y su "idiosincrasia" (rareza, particularidad) de acuerdo a entender su complejidad, y así, podrá narrar una historia sobre un sistema encerrado o impregnado, enfatizando la unidad y la totalidad del sistema, aunque confinando la atención de esos aspectos que son relevantes de los problemas de investigación en el tiempo (12).

Por lo expuesto, los EC cualitativos tienen algunas características especiales (21) que los investigadores deben tener en cuenta:

a. El contexto, donde cuatro capas o tipos en donde los fenómenos se involucran; el contexto inmediato (el presente aquí y ahora); el contexto específico (una perspectiva única e individual, que incorpora el pasado inmediato y las facetas significativas de una situación común); el contexto general (un marco de referencia de la vida general de los individuos), el metacontexto (una construcción social que representa una actitud social compartida y punto de vista).

b. Los bordes, en que los investigadores definen los bordes de la indagación, delinean el tema y los puntos de referencia -característica que distingue al enfoque cualita- 
tivo de EC ya que los límites o bordes son continuamente mantenidos con el énfasis en qué es y qué no es el caso-. Es decir, lo que sucede y seriamente importa dentro de esos límites (Emic) se considera vital y determina usualmente en qué consiste el estudio, y cómo se contrasta con otras clases de estudio donde las hipótesis o temas previamente centralizados por el investigador (Etic) son los que determinan el estudio.

c. El tiempo, los EC están orientados en el presente, ya que examinan experiencias contemporáneas más que eventos históricos. Aunque el investigador usa el dato histórico sobre las personas u organizaciones, se centraliza en el aquí y ahora, y es ésta una razón sobre la cual el EC se diferencia de las historias de vida (en los cuales sus crónicas unen y conectan las ocurrencias de vida de los grupos o individuos).

d. La intensidad, el EC usa una orientación intensiva del fenómeno bajo estudio. Hay una asociación cercana entre investigador y los participantes que ocurre en el tiempo, ya que el investigador se involucra por sí mismo en la situación o lugar, y recolecta la evidencia en forma intensiva para describir y/o explicar el caso.

Además, en la selección del caso también se debe considerar su priorización de caso único, y así la efectividad de esa particularización reemplaza la validez y la generalización, ya que lo que interesa es la profundización y el conocimiento global del caso (21). Por esto, Merriam (9) distingue tres características en su selección:

a. La particularidad del caso al señalar situaciones especiales, eventos, programas o fenómenos: el caso en sí es importante por lo que revela sobre el fenómeno y por lo que podría representar.

b. La descripción del caso, que significa que el producto final del caso es descripción única y densa del fenómeno bajo estudio. c. Lo heurístico del caso, significa que el caso ilumina el entendimiento del lector sobre el fenómeno en estudio, cuyo objetivo es descubrir un nuevo significado, confirmar lo que ya es conocido, o expandir una experiencia del lector, entre otras.

Posterior a la elección de un estudio de caso, Stake (12) señala que puede haber tres tipos de casos: a) el intrínseco, que resalta el entendimiento de un particular caso y su exclusividad (sin buscar que otros casos sean similares); b) el instrumental, el caso es usado para ilustrar el tema; y c) el de casos colectivos, el cual abarca más de un caso a estudiar con un entendimiento profundo de casos similares. Por ejemplo, se toma un caso simple como una organización, se separa en secciones que son luego analizadas y comparadas como un medio para obtener un conocimiento valioso sobre la organización como un todo.

Además, los EC cualitativos involucran una relación de los investigadores y su rol, como Stake, señala que en su proceso se debe: "circunscribir el caso y conceptualizar el objeto de estudio; seleccionar el fenómeno, temas, o tópicos; buscar los patrones de datos a desarrollar en los temas, triangular las observaciones claves para la interpretación; seleccionar métodos alternativos de interpretación; y desarrollar supuestos o generalizaciones sobre el caso" (12). Y agrega que los investigadores deben buscar la conexión sistemática entre las conductas observadas, las especulaciones, las causas y tratamientos y que para ello deben poseer ciertos requisitos como son las habilidades en su conducción y sentirse confortables frente a la ambigüedad y flexibilidad de la relación con lo inesperado, en más de una situación.

A continuación se muestra en el Diagrama 1 (adaptado por las autoras) el resumen de lo expuesto desde la selección del caso y los tipos resultantes de casos para su análisis interpretativo: 
Diagrama 1. Perspectiva de Estudio de Casos cualitativos.

\section{A. Perspectiva de Estudio de Casos}

(Adaptado por autores)

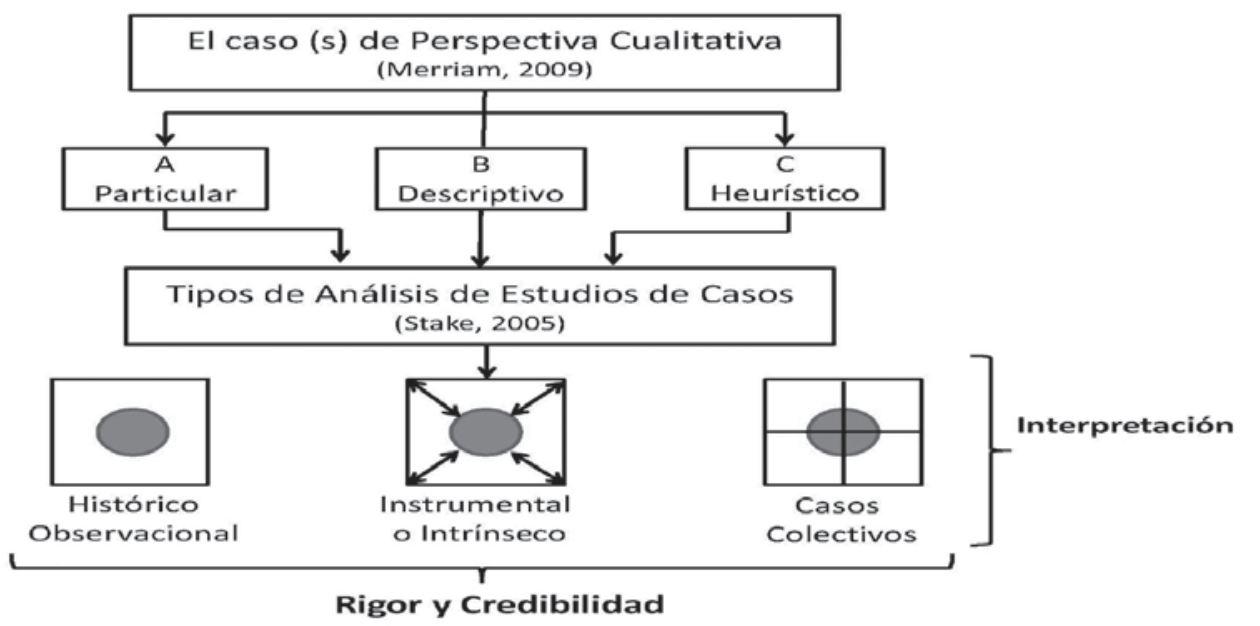

Adaptado por las autoras de: Merriam SB. Qualitative research. A guide to desing and implementation, 2009 (9) y Stake RE. Cualitative case studies, 2005 (12).

En síntesis, en un EC cualitativo el conocimiento que se aprende es diferente $(9,12)$, porque es más concreto; porque su conocimiento resuena con su propia experiencia que es vivida, real y sensorial; es más contextual, ya que la experiencia está enraizada en el contexto y este conocimiento se distingue desde el abstracto formal que deriva de otros diseños de investigación cualitativa, ya que tiene más desarrollo para la interpretación de los lectores al traer sus propias experiencias y su entendimiento y se basa más en la referencia poblacional (generalización naturalística) determinada por lo que los lectores tienen en mente como generalización $(22,23)$.

\section{B. Los Estudios de Casos como diseños me- todológicos}

Desde un nivel más simple los EC proporcionan cuentas descriptivas detalladas de uno o más casos cualitativos, y hasta un nivel más riguroso en un aislamiento experimental de algunos factores de una investigación cuan- titativa dentro de medios naturales. Como una perspectiva cualitativa los EC proporcionan una mirada con sus características y particularidades, lo que le da un marco de libertad mayor para descubrir lo inesperado, alguna regularidad, o disparidad que no anticipa una teoría (21). En cambio, en el diseño de investigación basado en EC se le concibe como: una mirada o un plan global del proceso por el cual la evidencia se recoge. El diseño, entonces, será la secuencia lógica que conecta los datos empíricos desde los inicios de la pregunta de investigación hasta sus conclusiones (10). El diseño incluirá los pasos según el enfoque, los métodos de recolección y análisis, tiempo, lugar y fuentes de datos que comprende lo siguiente $(3,6)$ :

1. Identificación del propósito y pregunta del estudio: Desde el contenido, organización, duración, recolección de los datos, y el tipo de evidencia serán dependientes del propósito de la indagación, y así el objetivo deberá ser enunciado explícitamente. 
2. Identificación de proposiciones teoréticas adecuadas al tipo de caso estudiado: Según el propósito del EC, las proposiciones teoréticas pueden guiar la indagación. Estas proposiciones se ven reflejadas en las preguntas de investigación, la revisión de literatura, y la expertez del investigador. Por ejemplo, si son estudios explicativos con testeo de hipótesis, la orientación teórica determinará el análisis $y$ el reexamen de las interpretaciones alternativas, o si son estudios exploratorios/descriptivos, involucrará en el dato mismo a las proposiciones teoréticas o marco de referencia determinado.

3. Determinación de las unidades de análisis: Como individuos, familias, organizaciones, eventos, etc., y del contexto en el cual la unidad de análisis sucede, deben ser diferenciados desde el principio del estudio. La claridad es imperativa para la recolección de los datos adecuados. Por ejemplo: si es un diseño de tipo holístico se examinará el fenómeno de interés como una totalidad, desde una perspectiva global (un grupo como un todo). Y si es un diseño de tipo embebido se examinarán las múltiples unidades y subunidades dentro del caso (aunque el estudio puede ser sobre una única entidad) (10).

4. Desarrollo de un protocolo de EC: Sirve de guía y mantiene el objetivo del estudio en la mira mientras se definen los procedimientos del campo, de recolección de datos y de análisis con un calendario preliminar para las actividades de recolección y las consideraciones de hechos inesperados (24). El protocolo identificará qué se obtendrá, con qué recursos, cuáles serán los datos recolectados, cuáles serán las fuentes de información u evidencia (quienes serán entrevistados, documentos examinados, qué observaciones se realizarán) y el plan de análisis y desarrollo de los reportes (25).

5. Decidir el diseño más apropiado de EC: Según Yin (26) hay dos diseños básicos en su conducción: el diseño de caso único y el diseño de caso múltiple (Figura 1). En el primero, si es un diseño de "caso único", habrá tres formas de ver el caso ya sea como crítico, extremo, o revelador. El caso crítico es apropiado cuando el investigador quiere testear una teoría bien formulada. El caso extremo (raro) es apropiado para aquellos en que no se identifican sus patrones. $Y$ un caso revelador es cuando se está privado de una situación porque no ha estado disponible a la indagación científica y entonces el EC ilumina sobre el fenómeno mal entendido. En el segundo, si el diseño está "embebido en los casos múltiples", las inferencias y las interpretaciones son diseñadas desde los grupos de casos. Este diseño es apropiado cuando el investigador está interesado en explorar el mismo fenómeno en una diversidad de situaciones o individuos.

Figura 1: Tipos y Diseños de Estudios de Casos.

\begin{tabular}{|c|c|c|}
\hline TIPOS DE ESTUDIO DE CASOS & Diseño Caso único & Diseño Caso Múltiple \\
\hline $\begin{array}{c}\text { HOLÍSTICO } \\
\text { Unidad de Análisis } \\
\text { SIMPLE }\end{array}$ & Tipo 1 & Tipo 2 \\
\hline $\begin{array}{c}\text { EMBEBIDO } \\
\text { Unidad de Análisis } \\
\text { MÚLTIPLE }\end{array}$ & Tipo 3 & Tipo 4 \\
\hline
\end{tabular}

Yin RK. Case study research: Design and methods, 2009 (26). 
6. Recolección de datos: Si bien la unidad de análisis puede ser solamente el caso único o un pequeño número de casos, el número de variables de interés en cada caso es generalmente amplio y deben ser examinadas ya que la evidencia puede venir de fuentes variadas: documentos, registros clínicos, entrevistas abiertas o estructuradas, observación directa y participante, entrevistas focales, y materiales (fotografía, filmes, videos) $(10,24,25)$.

7. Análisis de los datos: Según Yin (26), esta es la sección más difícil de realizar, ya que hay diferentes fórmulas, estrategias o técnicas existentes, y dependerá mucho ya sea del estilo del investigador/a, de su pensamiento si es riguroso o no, de la forma de presentación adecuada del proceso de análisis, o por último, de las consideraciones detalladas sobre interpretaciones alternativas. Por esta razón, Yin (26) señala dos estrategias: 1) desarrollo de una descripción del caso (sean puramente descriptivos y exploratorios) y 2) el empleo de una proposición teorética en el cual el es- tudio se basa. En general, en los casos múltiples (27) puede ser útil primero manejar los datos que analizan los casos individuales separadamente (análisis por dentro) y después todos los casos son chequeados en los elementos comunes y únicos (análisis cruzados) $(28,29)$. Actualmente, Yin $(26,30)$ propone cinco técnicas de análisis para una amplia variedad de contextos.

8. Reporte de EC: En general deben contener elementos como una explicación del problema o tema, una detallada descripción del contexto/lugar dentro del cual el fenómeno ocurrió, una completa delineación del proceso y el contexto y lugar que son relevantes al foco de la indagación, junto con una discusión de los resultados.

A continuación se muestra en el Diagrama 2 la estrategia de diseño de EC, adaptado por las autoras, en donde la pregunta determina cómo proceder en relación al tipo de diseño de casos, desde el punto de vista de Yin (10):

Diagrama 2. Estrategia basada en Estudio de Casos.

\section{B. Estrategia basada en Estudio de Casos}

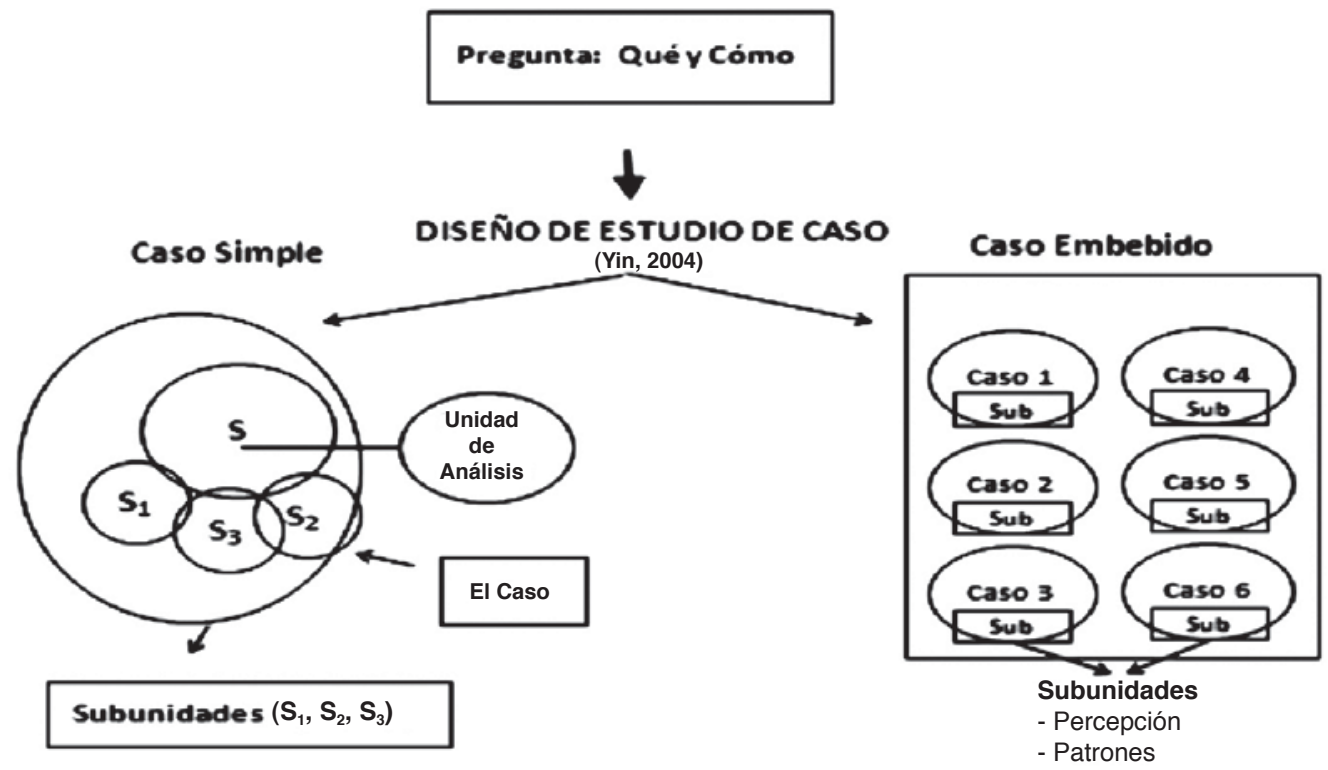

Yin RK. Case study research: Design and methods, 2004 (10). 


\section{CALIDAD Y USO DE LOS ESTUDIOS DE CASOS}

Los parámetros de calidad de validez y confiabilidad fundamentalmente son usados generalmente por los investigadores en la investigación cuantitativa dado la mirada empírica de los datos, otros investigadores buscan acomodar estos a criterios o protocolos para una generalización $(13,24)$. En perspectiva cualitativa, McGloin (31) ha usado un modelo de Fiabilidad de Lincoln y Guba (32) que consiste en credibilidad, dependibilidad, confirmabilidad y transferibilidad, y que sirven para chequear la calidad de los EC en los siguientes criterios: a) el valor de la verdad, es decir, cuán confiable es el investigador con la verdad encontrada en los hallazgos, al cumplir, ya sea, con la revisión de los grupos de pares, la reflexividad y la triangulación (33); b) la aplicabilidad del estudio, ya que en la conceptualización de caso único del EC, éste no puede ser representativo de una población, se puede entonces usar el término de generalización naturalista que expone cuán extenso es el reconocimiento de las entidades similares en y fuera del contexto $(34)$; c) la consistencia de los datos, si fuera realizado nuevamente el estudio aún con un grado de variabilidad, la fiabilidad puede obtenerse con un registro o grabación que asegure la precisión de la recolección de los datos; y d) la neutralidad, que se relaciona con cuán amplios son los hallazgos derivados de los participantes y de las condiciones de la investigación misma, y que no son el resultado de otras influencias o sesgos.

Otro punto ineludible es la importancia de la ética ya que como en todo estudio el investigador debe contar con reportes y registros para una forma de indagación segura y completa, tanto en la manipulación de datos, para no reportar datos contradictorios o con sesgo en la interpretación, y también en la protección del anonimato (interno y externo) de los participantes o sitios del es- tudio, de modo de asegurar la privacidad de los sujetos y contextos en donde se desarrolle el EC (9).

\section{CONSIDERACIONES FINALES DE SU USO Y CALIDAD INVESTIGATIVA}

Los ejemplos de desarrollo de los EC han sido más usados en el contexto investigativo de las ciencias sociales y en los últimos años en salud y enfermería de contexto anglosajón (1-5). Las posibilidades de uso de EC como perspectiva cualitativa o como diseño metodológico en contextos naturales ha sido usada en lugares como hospitales, organizaciones de salud, grupos o colectivos en donde el fenómeno de salud y/o de enfermería son complejos y difícil de separar entre las personas, su contexto, los bordes y en el tiempo. Ganganess y Yurkovich(25) ven a los EC apropiados para la enfermería práctica y su investigación en las poblaciones de forma de apoyar a la(s) comunidad(es), empoderar a las minorías, al usar recursos de datos múltiples, ya que aumenta la comprensión y el rigor. Esta variedad de ejemplos ha incluido la evaluación de programas y de políticas en salud, como señala Badger (35), quien realizó un estudio de multicasos como una estrategia de evaluación del sistema de salud en el área de salud mental al conducir casos con individuos, grupos, instituciones, y organizaciones. Bergen y While (36), por ejemplo, usaron un diseño de casos múltiples longitudinal para evaluar cuidados de enfermería de enfermos terminales en la comunidad. Resaltaron como los temas metodológicos más notorios revelados desde los EC son: la relación del fenómeno y el contexto, el grado de control del investigador sobre la definición del caso y los límites: la validez externa; la triangulación de métodos, y finalmente, la construcción de teoría. Más reciente, Zurmehly describió las prácticas de enfermería comu- 
nitaria y los factores asociados a la transición de la práctica clínica en contextos de cuidados en la comunidad, y usó el EC con entrevistas a 48 participantes enfermeras de diferentes programas de salud. Para su análisis, los datos fueron segmentados pero mantuvieron conexión como un todo, y así emergieron temas y patrones (37). En síntesis, Las enfermeras investigadoras al usar EC tienen un potencial en la práctica clínica, en organizaciones de atención primaria, escuelas, instituciones donde los procesos, patrones, situaciones de salud son adecuadas, con ambientes naturales y contemporáneos de salud y para dar respuestas a sus problemáticas $(38,39)$.

Actualmente, los Estudios de Casos son usados en una variedad de investigaciones $y$ se les reconoce su naturaleza flexible que pueden ahora involucrar paradigmas investigativos de naturaleza mixta (40), dado el supuesto de que la variabilidad de los casos afecta los temas o el fenómeno de interés, y puede ser así considerado como un puente entre éstos (41). Sin embargo, hay investigadores que asumen sólo estrategias y tradiciones cualitativas, Hewitt-Taylor (42) identifica una variedad de métodos de recolección de datos y discute sobre los principales temas relevantes y la importancia del investigador reflexivo para así producir finas descripciones, de tal forma que ilumine el significado y aumente el entendimiento del fenómeno. Otros como Tight (43) usan un EC mixto de un detallado examen de una muestra pequeña de un ítem de interés $y$ vista desde una perspectiva particular, de modo que el investigador se centralizó en un foco determinado de interés. Por último, Lloyd-Jones (2) usó el EC cuantitativo dentro de un experimento como una secuencia que proporcionó una oportunidad a examinar temas y su impacto. De este modo, dada la dispersión de posibilidades en EC, se han incorporado sugerencias de protocolos y representaciones esquemáticas para ayudar a los investigadores de EC (30).

\section{CONCLUSIÓN}

En síntesis, algunos investigadores seleccionarán EC cualitativos al considerar sus bases filosóficas más embebidas en las tradiciones cualitativas y la selección del caso será dada por su particularidad dentro del contexto, respondiendo así a una rica descripción detallada del caso en su contexto (44). Para otros, la pregunta será determinante para la selección de un diseño de EC y así seguirán sus pasos de recolección y análisis de datos, usando una variedad de fuentes que reportarán una amplia cantidad de información, es decir su distinción como diseño recae no tanto en los métodos empleados sino en las preguntas formuladas y su relación con el producto.

Enfermería en el medio sudamericano puede ampliar sus avances en el conocimiento propio y de salud al usar investigaciones de EC, teniendo presente estas dos aproximaciones investigativas en contextos y ambientes naturales en que suceden. Más específicamente, puede servir para mirar las acciones de enfermería desde el paradigma interpretativo-cualitativo en un contexto complejo como son los hospitales u organizaciones de salud, o por un sentido más pragmático cuantitativo de usar el EC dentro de un diseño que requiere una formulación de una pregunta de investigación indagada con diferentes métodos y responder así a la interrogante con más complejidad en ámbitos de salud naturales (45). Por último, una combinación de posibilidades de una investigación mixta teniendo en cuenta los aspectos metodológicos desde la validez o rigor que necesita estar presente ya sea para la generalización o para la fiabilidad.

\section{REFERENCIAS}

1. Antonhy S, Jack S. Qualitative case study methodology in nursing research: an integrative review. J Adv Nurs. 2009; 65(6), 
1171-1181.

2. Lloyd-Jones G. Design and control issues in qualitative case study research. Int J Qual Methods [Internet]. 2003[citado 04 de enero 2012]; 2(2): 1-19. Disponible en: http:www.ualberta.ca/ iiqm/backissues/2_2/pdf/lloyjones.pdf

3. Casey D, Houghton C. Clarifying case study research: examples from practice. Nurse Res. 2010; 17(3): 41-51.

4. Crowe S, Cresswell K, Robertson A, Huby G. Avery A, Sheikh A. The case study approach. BMC Med Res Methodol. 2011; 11: 100 .

5. Walshe, C. The evaluation of complex interventions in palliative care: An exploration of the potential of case study research strategies. J Palliat Med. 2011; 25(8): 744-781.

6. Pelgram, A. What is case study research. Nurse Res. 1999/2000, 7(2): 5-16.

7. Gerring J. Case studies research. Principles and practices. Cambridge University Press: NY, 2007.

8. Johansson R. Case study methodology. International Conference Methodologies in Housing Research; 22-24 Setiembre 2003; Stockholm: Royal Institute of Technology, International Association of People-Environment Studies; 2003. 14 p.

9. Merriam SB. Qualitative research. A guide to design and implementation. San Fco, CA: Jossey-Bass; 2009.

10. Yin RK. Case study research: Design and methods. 3a. ed. Thousands Oaks, CA; Sage. 2004.

11. Stake RE. The art of case study research. Thousands Oaks, CA: Sage; 1995.

12. Stake, RE. Qualitative case studies. En: Denzin NK, Lincoln YS, editores. The handbook of qualitative research. $3 \mathrm{a}$ ed. Thousand Oaks, CA: Sage; 2005. p. 44366.

13. Verschuren PT. Case study as a research strategy: some ambiguities and opportunities. Int J Soc Res Methodol. 2003; 6(2): 121-139.
14. Jones C, Lyons C. Case study: design, method, or comprehensive strategy? Nurse Res. 2004; 11(3): 70-76.

15. Baxter P, Jack S. Qualitative case study methodology: study design and implementation for novice researchers. Qual Report. 2008; 13(4): 544-59.

16. Reyes P, Hernández A. El estudio de casos en el contexto de la crisis de la modernidad. Cinta Moebio. 2008: 32; 70-89.

17. Mariano C. Case Study: The method. En: Munhall PL, Oiler Boyd C, editores. Nursing Research: A qualitative perspective. Boston, MA: Jones and Barlett Publishers; 1999. p. 233-260.

18. Tesch LR. Qualitative Research: Analysis types and software tools. NY: Falmer; 1990.

19. Creswell JW. Qualitative inquiry and research design. Choosing among five traditions. $2 \mathrm{a}$ ed. Thousand Oaks, CA: Sage; 2007.

20. Neiman G, Quaranta G. Los estudios de casos en la investigación sociológica. En: Vasilachis de Gialdino, editor. Estrategias de investigación cualitativa. Barcelona, ES: Gedisa Editorial; 2006. 212-37 p.

21. Hinds P, Chaves D, Cypess S. Context as a source of meaning and understanding. En Morse J, editor. Qualitative health research. Newsbury: CA: Sage; 1992. p. 3142.

22. Meyer C. A case in case study methodology. Field Methods. 2001; 13(4): 329-352.

23. Ghesquiere P, Maes B, Vandenberghe R. The usefulness of qualitative case studies in research on special needs education. Int. J Disab Devel Educ. 2004; 51(2): 171184.

24. Tellis W. Application of a case study methodology. Qual Report. 1997; 3(3): 1-17.

25. Ganganess J, Yurkovich E. Revisiting case study as a nursing research design. Nurse Res. 2006, 13(4): 7-18.

26. Yin RK. Case study research: Design and methods. 4a. ed. Thousands Oaks, CA; Sage. 2009. 
27. Miles MB, Huberman AM. Qualitative data analysis; a sourcebook of new methods. B. Hills, CA: Sage; 1984.

28. Hopwood N. Research design and methods of data collection and analysis: researching students' conceptions in a multiple-method case study. J Geog Higher Educ. 28(2): 347-353.

29. Rosemberg JP, Yates PM. Schematic representation of case study research designs. J Adv Nurs. 2007; 60(4): 447-452.

30. Yin RK. Application of case studies research. 3a Ed. Thousand Oaks, CA: Sage: 2012.

31. McGloin S. The trustworthiness of case study methodology. Nurse Res. 2008; 16(1): 45-55.

32. Lincoln Y, Guba E. Naturalistic Enquiry. Thousand Oaks, CA, Sage; 1985.

33. Ryan-Nicholls KD, Wills C. Rigor in qualitative research: mechanisms for control. Nurse Res. 2009; 16(3): 76-85.

34. Houghton C, Casey D, Shaw D, Murphy $\mathrm{K}$. Rigour in qualitative case-study research. Nurse Res. 2013; 20(4): 12-17.

35. Badger T. A case study exemplar: health outcomes of people with serious mental illness. En: Mundhall P. Nursing Research. A qualitative perspective. $4^{\text {a }}$ ed. Ontario, Canadá: Jones \& Bartlett; 2007.

36. Bergen A, While A. A case for case studies: exploring the use of case study design in community nursing research. J.Adv Nurs. 2000; 31(4): 926-934.

37. Zurmehly J. A qualitative case study review of role transition in community nursing. Nurs Forum. 2007; 42(2): 162170.

38. Sangster-Gormley E. How case-study research can help to explain implementation of the nursing practitioner role. Nurs Res. 2013, 20(4): 6-11.

39. Urra E. Struggling to achieve professional recognition: A case study of nursing in Chile. Hamburgo: Lambert, 2010.

40. Creswell JW. Research design: qualitative, quantitative, and mixed method approaches. Sage, CA: 2009.

41. Luck L, Jackson D, Usher K. Case study: a bridge across paradigms. Nurs Inq. 2006; 13: 103-109.

42. Hewitt-Taylor J. Case study: an approach to qualitative enquiry. Nurs Stand. 2002; 16(20): 33-37.

43. Tight M. The curious case of case study: a viewpoint. Int J Social Res Method. 2010; 13(4): 329-339.

44. Taylor R. Case study research in context. Nurs Res. 2013, 20(4): 4-5.

45. Welford C, Murphy K, Casey D. Desmystifying nursing research terminology: Part 2. Nurs Res. 19(2), 29-35. 